\section{Illuminated artificial orbit for the training of vitreoretinal surgery in vitro}

CE Uhlig and $\mathrm{H}$ Gerding

have been reported previously. ${ }^{1-15}$ However, there is still a demand for didactic devices teaching experimental handling and controlling of specific problems to novice vitreoretinal surgeons. An optimal device would have the following characteristics: (i) stabilization of the eyeball even during long-term interventions; (ii) prevention of the stretching of the eyeball during scleral depression and tractions; (iii) illumination of the vitreoretinal segment homogenously without dazzling the operator; and (iv) accentuation of mechanical alterations of the vitreal structures owing to traction movements of the surgeon.

An artificial orbit with diascleral illumination that incorporates the above characteristics has been developed for experimental and didactic use during vitreoretinal interventions in vitro. via the light guide into the cylind and through the eye support transsclerally into the eye.

Results The device illuminates the retina and the vitreous body sufficiently. It accentuates the transparent appearance of the retina and makes the three-dimensional structure more visible, for example, in case of retinal detachment. Subretinal tissue can be visualized and differentiated more precisely. Conclusions The device is useful, in the teaching of residents and in experimental vitreoretinal surgery.

Eye (2004) 18, 183-187. doi:10.1038/

sj.eye. 6700596

Keywords: artificial orbit; eye-support; training; experimental microsurgery; vitreoretinal microsurgery

\section{Introduction}

Teaching and training of microsurgery procedures are main constituents of the medical education of residents in ophthalmology. As mechanical assistance is necessary in vitro, various devices for use during the training of different diagnostic or microsurgery procedures

\section{Materials and methods}

Experiments were performed with various light-sources, including normal incandescent bulbs and halogen lamps, and with different designs of eye supports. The intensity and homogeneity of illumination were compared, as well as the method of eyeball fixation. Devices with differing proportions and dimensions were also tested.

The components of the final prototype

(Figure 1a) are as follows: a high-intensity point light source (Zeiss, Germany) using a 100-W halogen lamp (nr. 6834, Philips, Germany), a transformer for supplying the light source (5.4 or 11.5 V output, Spindler \& Hoyer, Göttingen, Germany), a flexible light guide (0.5-cm diameter fibre), a cylinder $(4.2 \mathrm{~cm}$ in height, $3.4 \mathrm{~cm}$ in diameter) with a mirror inside (24 $\mathrm{mm}$ in diameter, angled $45^{\circ}$ relative to the base), and fixed to a plate $(6.0 \mathrm{~cm}$ in diameter, $1.0 \mathrm{~cm}$ in height), and a replaceable eye support with an adapted ring (Figures $1 \mathrm{~b}$ and 2). The transformer can be used to adjust the light intensity from the point source according to the scleral thickness. The light is fed into the
University Eye Hospital

Muenster, Germany

Correspondence:

CE Uhlig

University Eye Hospital

Muenster

Domagkstrasse 15

48129 Muenster, Germany

Tel: + 492518356004

Fax: +492518356003

E-mail: uhligc@uni-

muenster.de

Received: 2 December 2002 Accepted in revised form: 21 March 2003

Financially supported by the BMFT, German National Ministry of Research and Technology, EPI-RET grant (01 IN 501 D)

Proprietary interests: Nil Commercial interests: Nil 

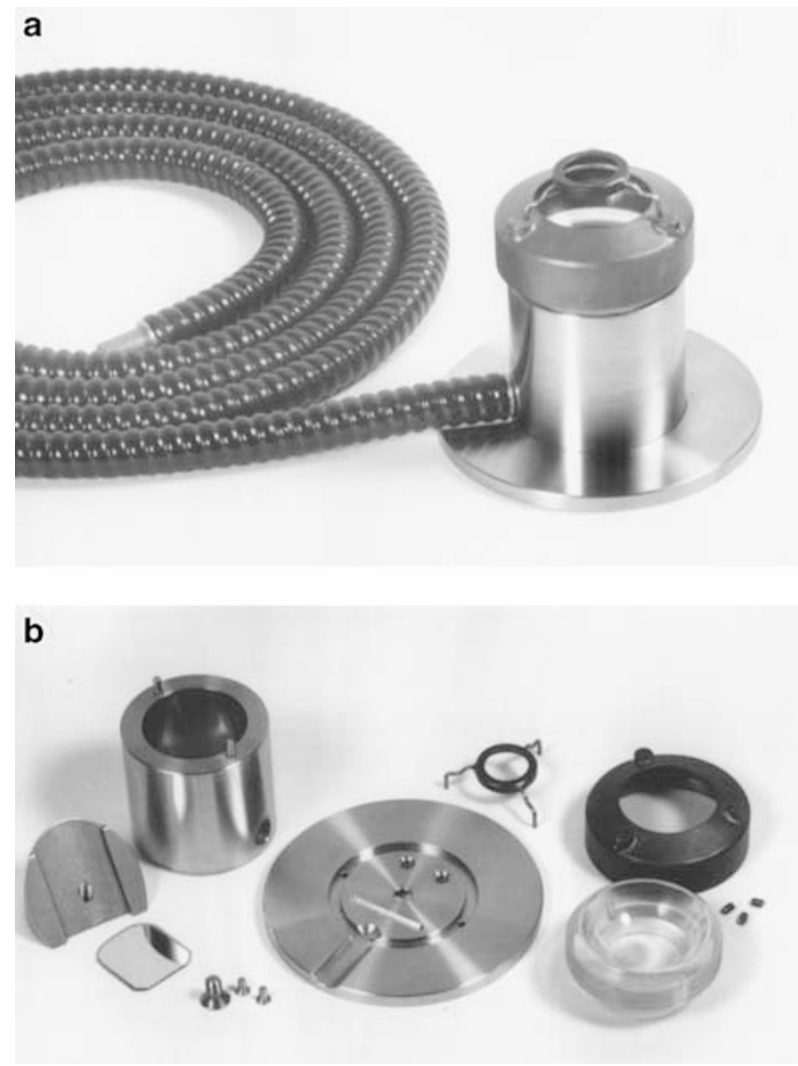

Figure 1 (a) Artificial orbit with flexible light guide. (b) Principal components of the device.

cylinder using the light guide, and from there via the mirror to the eye support. The eye support is made of transparent polymethylmethacrylate, so that the light penetrates the sclera and illuminates the eyeball. The eye support is fitted simply into the cylinder and is thus replaceable, and it is sandblasted on its underside while its upperside is concave and polished, with dimensions appropriate for the individual eye under investigation. A metal ring is screwed onto the top of the eye support to additionally fixate the eyeball. A further ring for using a Kilp-contact-lens is positioned on top. Each component of the device is fixed to another or to the base unit with screws.

\section{Results}

In vitro examinations were carried out with human, rabbit, and porcine eyes obtained the first hours post mortem. The illumination intensity when using incandescent or halogen lamps up to $35 \mathrm{~W}$ was not adequate for human or porcine eyes, but $100 \mathrm{~W}$ lamps were found to be sufficient. Transformer outputs between 5.4 and $11.5 \mathrm{~V}$ were finally chosen for rabbit and porcine eyes, 12 and $96 \mathrm{fL}$ respectively, and human eyes
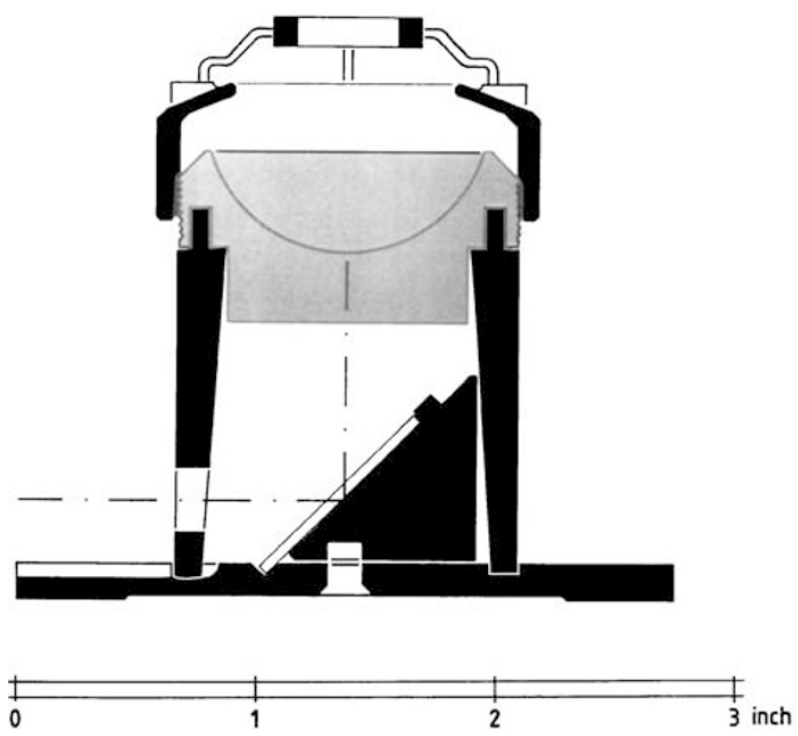

Figure 2 Sectional drawing of the device.

required an intermediate light intensity. Although the device uses a light guide with a small diameter $(0.5 \mathrm{~mm})$, the illumination was sufficient for all tested species, most especially for the human and rabbit eyes: scleral transillumination in porcine eyes was sufficient but not very intense even with a brightness of $96 \mathrm{fL}$ owing to the scleral thickness and pigmentation.

The use of different eye supports enables the quasisimultaneous preparation and investigation of eyes from different species (ie rabbit, porcine, or human eyes in the study). The ring adapter stabilizes the eyeball even during long-term interventions. The eye support prevents the stretching of the eyeball during scleral depression and traction. The diascleral illumination combined with an intraocular light source enables the resident to identify mechanical alterations of the vitreal structure owing to traction movements. The upper ring adapter allows the use of a Kilp-contact-lens without the help of an assistant (Figure 3). The flexible light guide and the inclusion of the mirror inside the cylinder allow the device to be short, resulting in easy and comfortable handling, and illumination of the posterior pole, the vitreous body, and the lens.

As the diascleral light illuminates the retina and the vitreous, it allows the resident to enter the eyebulb with the vitreo-cutter without problems usually resulting from the additional handling of an intraocular light source. Illuminating the vitreoretinal space of a porcine eye alone with an intraocular light source results in a whitish appearance obscuring retinal and subretinal details of the fundus (Figure 4a). Without diascleral illumination, retinal vessels and the internal surface of the retina are the only landmarks for the resident (Figure 4b). Instead, 


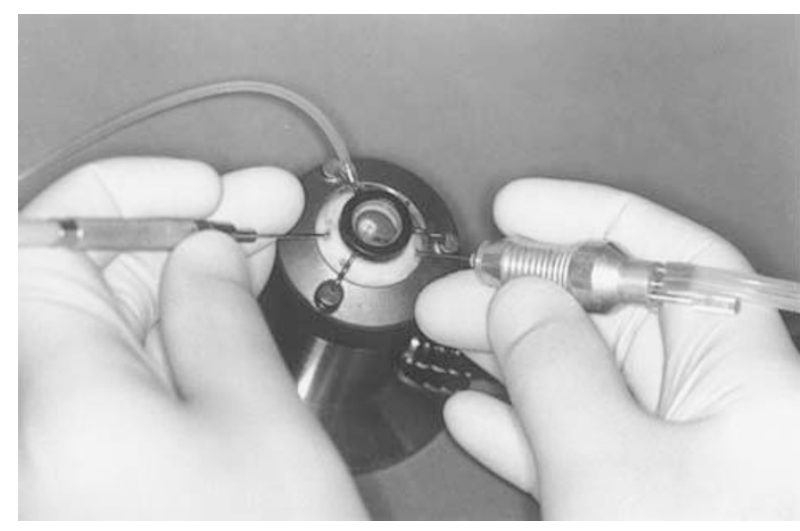

Figure 3 Artificial orbit with diascleral illumination in use with a porcine eye.
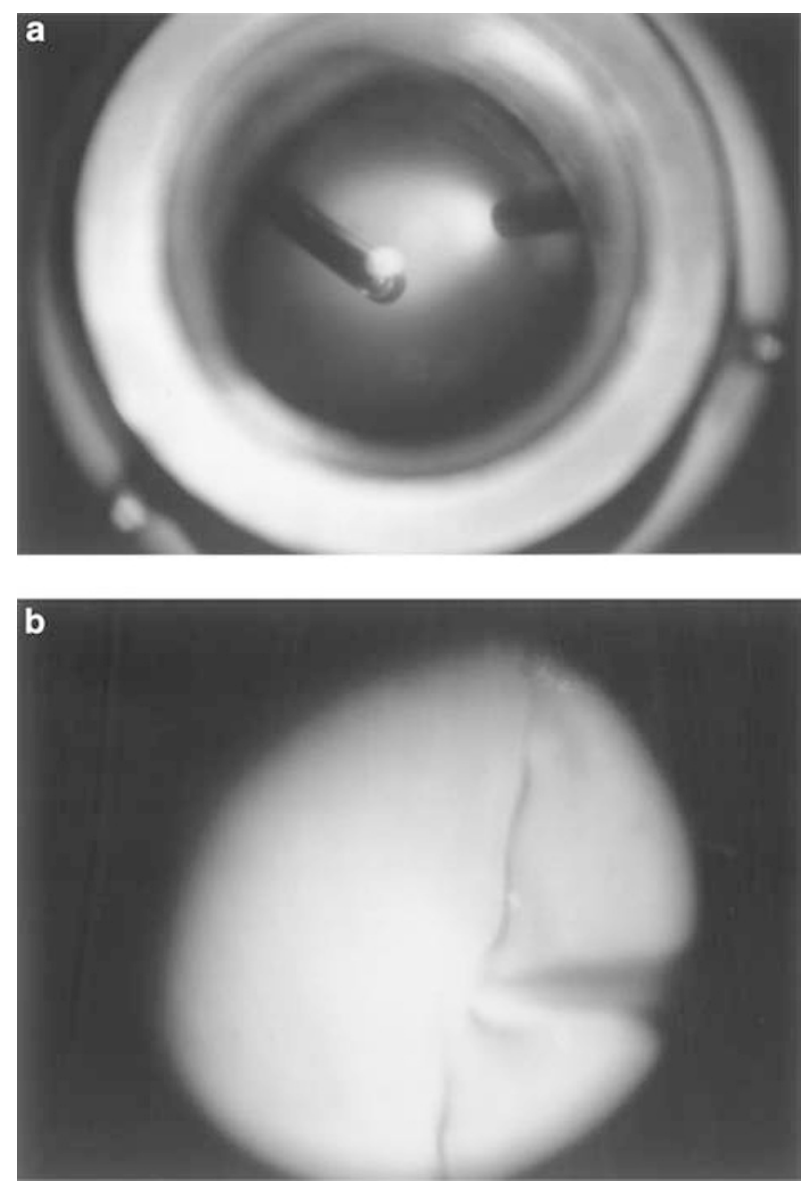

Figure 4 ( $a$ and $b$ ) Porcine retina in vitro illuminated with an intraocular light source.

the use of the diascleral light enables to see the retinal tissue with its transparent appearance and the underlying uveal vessel architecture. The diascleral illumination accentuates the retina as a three-
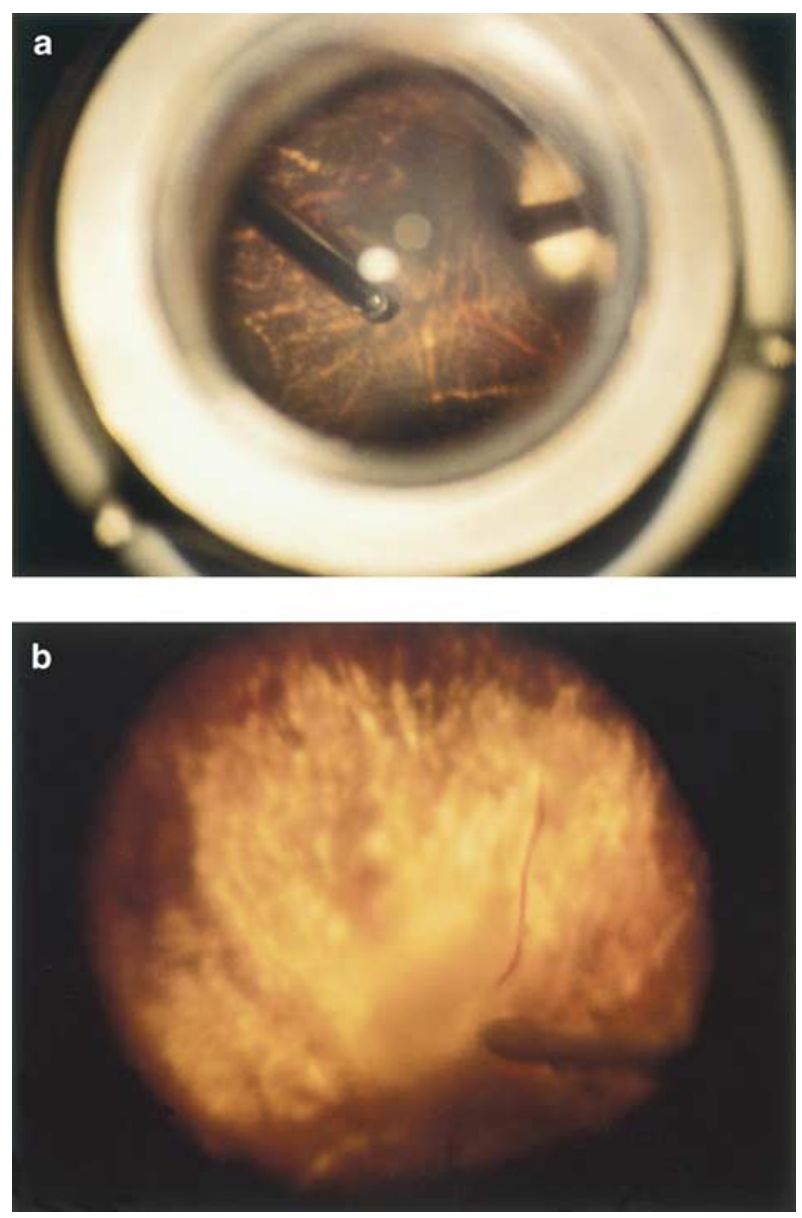

Figure 5 ( $a$ and $b$ ) Porcine retina in vitro illuminated diasclerally.

dimensional tissue and allows visualization of the subretinal space in case of retinal elevation (Figure 5a and b). As an additional means of an intraocular light source, the diascleral illumination makes retinal defects like holes or detachments easier to be seen (Figure 6a and $b$ ).

The diascleral illumination highlights the posterior lens capsule and trains the resident to avoid touching the lens. An axial or coaxial light from a microscope can be used temporarily if the anterior capsule needs to be accentuated.

\section{Discussion}

The demand for didactic methods in ophthalmology has encouraged the development of various instruments for the training of in vitro microsurgical procedures and techniques. Devices have been proposed for training in the use of laser application of the anterior and posterior segment $t^{4,6,7,9,10,12}$ and the use of eye models in indirect 

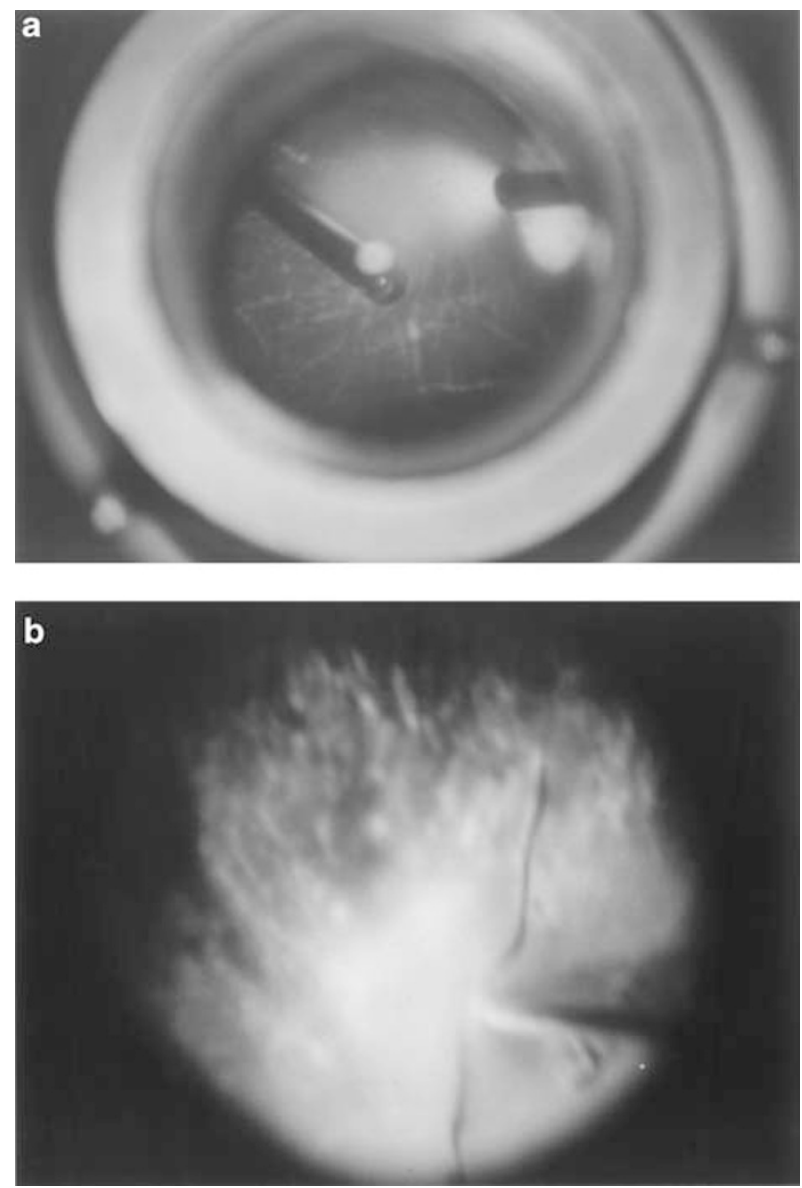

Figure 6 (a and b) Porcine retina in vitro illuminated diasclerally and with an intraocular light source.

ophthalmoscopy. ${ }^{1,2,8,10,12}$ Computer techniques, such as those involving the display of images stored on videodiscs ${ }^{16}$ or surgery simulators ${ }^{13-15}$ have been of additional use. However, using didactic methods involving artificial eye models or stored images is quite different from experimenting on real tissue. This has led to the developed devices for holding the eyeball for use in the training of in vitro microsurgical techniques. ${ }^{3,5}$ These devices are especially useful for the training of keratoplasty, glaucoma, and cataract procedures. The eyeholder from Arentsen and Duran ${ }^{3}$ can be illuminated, by positioning a light source just under the organ, but in vitro studies in our laboratories with comparable incandescent and halogen bulbs up to $35 \mathrm{~W}$ did not sufficiently illuminate human or porcine eyes, and the shape and pigmentation of the eyes meant that the illumination was not uniform. We conclude that the device from Arentsen and Dodaro, as well as that from Spitznas, ${ }^{5}$ can be used for practising general vitreoretinal techniques, but that they are not suitable for novice vitreoretinal microsurgeons because of insufficient illumination of the subretinal space and the threedimensional structure of the retina, and they do not allow the quasi-simultaneous study of eyes from different species.

A new artificial orbit consisting of a high-intensity point light source, a transformer, a flexible light guide, and a replaceable eye support for diascleral illumination of eyeballs has been designed and tested. The instrument accentuates the retina and the subretinal space. The method of diascleral illumination is different from that normally used in surgery. However, in comparison to endoillumination or to a light from a microscope, it gives in vitro a more natural image. It enables the resident to use the Kilp-contact-lens without further personal assistance. Different-sized eye supports allow the use of eyes from several species. When the additional aim of the Kilp-contact-lens is not necessary, it is also possible to use human eyes, following corneal removal for transplantation, with a specific keratoprosthesis. ${ }^{11}$

The eye support with retrograde illumination also produces good results during microsurgery of the posterior chamber, highlighting the posterior lens capsule during lensectomy, which is a procedure of high risk when performed by novice cataract surgeons.

The artificial orbit is a useful aid in didactic demonstrations and experimental vitreoretinal interventions in vitro.

\section{Acknowledgements}

We would like to thank J Boes, W Ruck, and L Scheidgen for manufacturing the final artificial-orbit prototype.

\section{References}

1 Bartner H, Paton D. A model eye for instruction in indirect ophthalmoscopy. Ann Ophthalmol 1969; 1: 42-46.

2 Colenbrander A. Simulation device for ophthalmoscopy. Am J Ophthalmol 1972; 74: 738-740.

3 Arentsen J, Duran M. Stereotaxic device for experimental eye surgery. Invest Ophthalmol 1976; 15: 34-36.

4 Whitacre MM, Mainster MA. Photographic slides for teaching laser photocoagulation. Am J Ophthalmol 1987; 103: 590-591.

5 Spitznas M. A training device for intraocular surgery (Ein Übungssockel für intraokulare Eingriffe am Auge). Klin Monatsbl Augenheilkd 1976; 168: 435-436.

6 Shammas AV, Minckler DS. Autopsy eye model for laser trabeculoplasty and iridectomy. Am J Ophthalmol 1986; 102: 664-665.

7 Minckler DS, Gaasterland D, Erickson PJ. Improved, reusable, autopsy eye model container for laser trabeculoplasty and iridectomy. Am J Ophthalmol 1992; 113: 341-342.

8 Chew C, Gray RH. A model eye to practice indentation during indirect ophthalmology. Eye 1993; 7: 599-600. 
9 Oram O, Gross RL, Severin TD, Feldmann RM, OrengoNania S. A human cadaver eye model for anterior and posterior segment laser applications. Ophthalmic Surg 1994; 25: 449-451.

10 Dodaro NR, Maxwell Jr DP. An eye for an eye. A simplified model for teaching. Arch Ophthalmol 1995; 113: 824-826.

11 Uhlig C, Gerding H. A keratoprosthesis for training and experimental-surgical use of human donor eyes following removal of the corneal transplant (Keratoprothese für die experimentell-operative Nutzung von Spenderaugen nach Hornhautentnahme). Ophthalmologe 1997; 94: 501-505.

12 Uhlig CE, Gerding H. A dummy orbit for training in diagnostic procedures and laser surgery with enucleated eyes. Am J Ophthalmol 1998; 126: 464-466.
13 Hikichi T, Yoshida A, Igarashi S, Mukai N, Harada M, Muroi $\mathrm{K}$ et al. Vitreous surgery simulator. Arch Ophthalmol 2000; 118: 1679-1681.

14 Wagner C, Schill M, Hennen M, Männer R, Jendritza B, Knorz MC et al. Virtual realities for the training of eye surgeons (Virtuelle Realitäten für die augenchirurgische Ausbildung). Ophthalmologe 2001; 98: 409-413.

15 Verma D, Wills D, Verma M. Virtual reality simulator for vitreoretinal surgery. Eye 2003; 17: 71-73.

16 Folberg R, Dickinson LK, Christiansen RA, Huntley JS, Lind DG. Interactive videodisc and compact disc-interactive for ophthalmic basic science and continuing medical education. Ophthalmology 1993; 100: 842-850. 